\title{
An influence of normal stress and pore pressure on the conditions and dynamics of shear crack propagation in brittle solids
}

Cite as: AIP Conference Proceedings 1783, 020207 (2016); https://doi.org/10.1063/1.4966501 Published Online: 10 November 2016

Evgeny V. Shilko, Sergey G. Psakhie, and Valentin L. Popov

ARTICLES YOU MAY BE INTERESTED IN

Mathematical model and numerical simulation of slow deformation waves in the earth's crust structural elements

AIP Conference Proceedings 1783, 020146 (2016); https://doi.org/10.1063/1.4966439

Paradoxes of high and low velocities in modern geodynamics

AIP Conference Proceedings 1783, 020145 (2016); https://doi.org/10.1063/1.4966438

Blow-up regimes in failure of rock specimens

AIP Conference Proceedings 1783, 020215 (2016); https://doi.org/10.1063/1.4966509
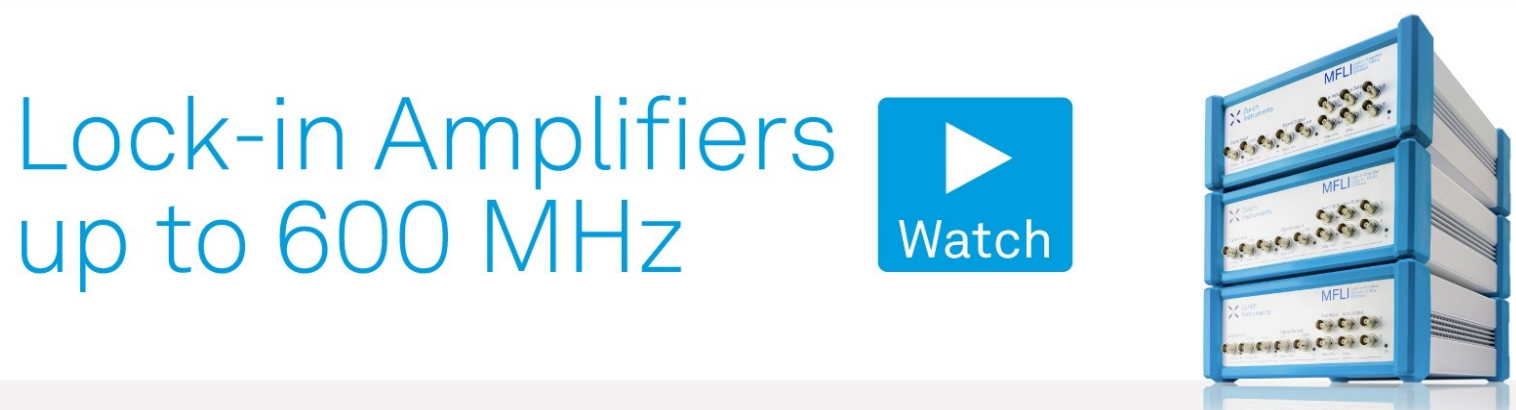


\title{
An Influence of Normal Stress and Pore Pressure on the Conditions and Dynamics of Shear Crack Propagation in Brittle Solids
}

\author{
Evgeny V. Shilko ${ }^{1,2, a)}$, Sergey G. Psakhie ${ }^{1,3, b)}$, and Valentin L. Popov ${ }^{4, c)}$ \\ ${ }^{1}$ Institute of Strength Physics and Materials Science SB RAS, Tomsk, 634055 Russia \\ ${ }^{2}$ National Research Tomsk State University, Tomsk, 634050 Russia \\ ${ }^{3}$ National Research Tomsk Polytechnic University, Tomsk, 634050 Russia \\ ${ }^{4}$ Berlin University of Technology, Berlin, 10623 Germany \\ a) Corresponding author: shilko@ispms.tsc.ru \\ ${ }^{b)}$ sp@ispms.tsc.ru \\ c)v.popov@tu-berlin.de
}

\begin{abstract}
The paper is devoted to the study of the influence of crack-normal stress on the shear strength of the brittle material with initial crack and the geometrical condition of acceleration of dynamically growing crack towards the longitudinal wave speed. We considered elastic-brittle permeable materials with nanoscale pore size. We have shown that pore fluid in nanoporous brittle materials influences mainly the condition of shear crack propagation transition from conventional sub-Rayleigh regime to supershear one. The results of the study make it possible to assess the ability of initial cracks in brittle materials to develop in supershear regime under the condition of confined longitudinal shear.
\end{abstract}

\section{INTRODUCTION}

The questions concerning brittle fracture of porous (including nanoporos) solids are widely discussed during a decades [1-3]. A special attention is paid to the analysis of the conditions of initiation and regimes of dynamic propagation of longitudinal shear cracks. Considerable interest in the study of the conditions under which a longitudinal shear crack is able to propagate in supershear regime, is concerned with its close connection with the problems of initiation and regimes of dynamic slip in contact zones, dynamics of earthquakes and so on [4-6].

Among porous materials we can distinguish a subclass of materials with nanoscale pore size. A pronounced feature of nanoporous brittle solids is an important role of adsorption effects. Fluid filtration in nanoporous solids is possible on condition that pore pressure exceeds a threshold value. Typical value of threshold pore pressure for nanosized pores $\left(d_{\text {pore }}<0.01 \mu \mathrm{m}\right)$ may reach several tens of megapascals, that is comparable to the material cohesion. Therefore, at relatively low mean stresses the mechanical deformation of fluid saturated nanoporous materials is not accompanied by fluid redistribution and determined by local pore pressure.

In some recent papers the authors analyzed the key peculiarities of the dynamics of longitudinal shear crack propagation in brittle solids [7, 8]. We showed that dynamic growth of mode II crack is accompanied by the formation of an elastic vortex ahead of the crack tip. The elastic vortex is a dynamic shear stress concentrator propagating at a shear wave velocity ahead of the crack. Shear stress growth in the elastic vortex provides a possibility of crack acceleration towards the anomalously high velocities up to the longitudinal wave speed. However, this study didn't touch upon the issues of the influence of applied crack-normal stress on the functional form and threshold (critical) values of the criteria determining the onset of dynamic propagation of mode II crack in brittle materials and the ability of an initial crack to propagate in supershear regime. These issues are directly related to the problem of an influence of pore pressure on the conditions and dynamics of supershear crack propagation in nanoporous solids. The present paper is devoted to the numerical study of above mentioned problems. 


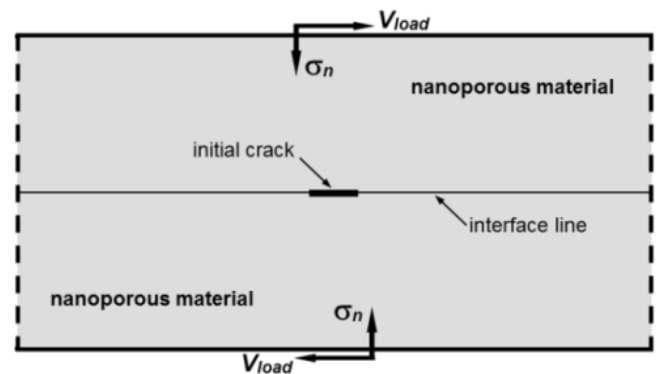

FIGURE 1. Schematic and the loading scheme of the two-dimensional model slab made from nanoporous brittle material

\section{PROBLEMS STATEMENT}

The study was carried out by means of movable cellular automaton (MCA) based numerical simulation. The MCA method is a representative of the group of discrete element methods, namely of the subgroup of distinct elements [9]. The study of the dynamic growth of longitudinal shear cracks was carried out in the framework of the macroscopic consideration of the material. We considered a model nanoporous $\left(d_{\text {pore }}<0.01 \mu \mathrm{m}\right)$ macroscopically isotropic linear-elastic (brittle) material with porosity $10 \%$. Typical elastic and strength parameter of porous $(\sim 10 \%)$ sandstones were used as parameters of the model material. The pore structure and interstitial fluid (water) were implicitly taken into account through the elastic parameters of porous solid and interstitial fluid. The "macroscopic" influence of pore fluid pressure on the stress state of the solid skeleton was described using Biot's model of poroelasticity. To take into account dependence of material shear strength on mean stress we used two-parametric fracture criterion of Drucker and Prager with effective mean stress $\sigma_{\text {mean,eff }}=\sigma_{\text {mean }}+p_{\text {pore }}\left(\sigma_{\text {mean }}\right.$ is mean stress determined by external load, $p_{\text {pore }}$ is pore pressure) instead of $\sigma_{\text {mean }}$ in fracture criterion for dry material.

The numerical study was carried out with use of the two-dimensional model slab (Fig. 1) and under the condition of plane strain. The slab consists of two parts bonded through the interface. Plates have the same properties and are considered as a linear elastic and high-strength, while interface has much smaller strength. A small initial crack was assigned at the interface. In the study we analyzed dynamic growth of an initial crack along the interface.

Preliminary stress state of the sample was set by applying vertical compressive load to the upper and lower surfaces. Subsequent longitudinal shear was realized by displacing the upper and lower borders of the pre-stressed sample in the horizontal direction at low velocity (vertical positions of the borders remain fixed). Such loading conditions correspond to confined longitudinal shear. Note that one of the main characteristics of confined shear is the value of applied crack-normal (and interface-normal) stress $\sigma_{\mathrm{n}}$, which determines the value of means stress in the sample. Periodic boundary conditions were applied in horizontal direction (Fig. 1).

Note that in the first stage of shear deformation the slab is deformed without fracture and accumulates elastic strain energy. When the shear load reaches some limiting value (shear strength $\tau_{0}$ of the sample with the initial crack), the crack starts to propagate dynamically. Note that $\tau_{0}$ depends on geometrical parameters of the initial crack and amounts to a portion of shear strength of intact interface at applied crack-normal stress $\sigma_{\mathrm{n}}$.

\section{SIMULATION RESULTS AND DISCUSSION}

The results of the simulation showed common dynamics of unstable crack growth in conventional sub-Rayleigh regime for both dry and water-saturated nanoporous brittle materials. In particular, a key feature of the initial phase of dynamic shear crack growth is the formation of the collective elastic vortex-like motion of material particles (hereinafter called an elastic vortex) in the vicinity of the moving crack tip [7]. Elastic vortex is associated with concentration of shear stresses in the elliptical region, which is situated in the frontal part of the vortex. The magnitude of shear stresses in the elastic vortex gradually increases during the course of propagation until vortex detaches from the crack (elastic vortex propagates faster than sub-Rayleigh crack) [7].The maximum magnitude of shear stress is achieved before vortex detaching from the crack. This magnitude depends on the density of elastic shear strain energy accumulated in the system during the course of stable shear deformation (before the moment of initial crack growth start). If the value of accumulated elastic shear strain energy is enough to provide reaching the 
interface strength by the shear stress in the vortex (this value is called threshold or critical shear strain energy), then the secondary rupture appears at the interface ahead of the growing crack, in the frontal part of the elastic vortex. This secondary rupture is able to propagate at a velocity higher than the shear wave speed (in supershear regime).

Simulation results have shown that energy criterion of crack acceleration to intersonic velocities at a constant value of applied crack-normal stress $\sigma_{\mathrm{n}}$ is a characteristic magnitude of accumulated elastic shear strain energy. This magnitude can be characterized by the value of shear strength $\tau_{0}$ of the slab with an initial crack. Shear strength $\tau_{0}$ is a unique function of dimensionless geometrical parameter of the initial crack $P=L / D$ (where $L$ is an initial crack length and $D$ is effective thickness of an initial crack) at a constant value of $\sigma_{\mathrm{n}}$. Under the condition of confined shearing the shear strength $\tau_{0}$ is an increasing function of the value of crack normal stress $\sigma_{\mathrm{n}}$. We have built a general functional form of the dependence of the shear strength $\tau_{0}$ of the slab with an initial crack on the value dimensionless geometrical crack parameter $P$ at applied crack normal stress $\sigma_{\mathrm{n}}$ (Fig. 2a):

$$
\tau_{0}=\tau_{\text {is }}\left(\sigma_{\mathrm{n}}\right) \sqrt{\frac{1}{1+\alpha\left(\sigma_{\mathrm{n}}\right) P}\left(1-\left(\frac{\tau_{\infty}\left(\sigma_{\mathrm{n}}\right)}{\tau_{\text {is }}\left(\sigma_{\mathrm{n}}\right)}\right)^{2}\right)+\left(\frac{\tau_{\infty}\left(\sigma_{\mathrm{n}}\right)}{\tau_{\text {is }}\left(\sigma_{\mathrm{n}}\right)}\right)^{2}},
$$

where $\tau_{\text {is }}$ is the shear strength of intact interface at the assigned value of $\sigma_{\mathrm{n}}, \tau_{\infty}$ is the shear strength of the interface with semi-infinite crack, $\alpha$ is a dimensionless coefficient, which depends on material properties and $\sigma_{\mathrm{n}}$.

Main differences between curves $\tau_{0}(P)$ at different values of $\sigma_{\mathrm{n}}$ are connected with increasing the shear strength of the interface with semi-infinite crack as $\sigma_{\mathrm{n}}$ increases. This can be explained by complex stress state of tip of the initial crack under the condition of longitudinal shear. This stress state includes crack normal tensile stress. Contribution of tensile stress to stress state in the vicinity of the crack tip decreases with increase in the normal stress $\sigma_{\mathrm{n}}$ (crack opening displacement is reduced down to zero, and at large values of $\sigma_{\mathrm{n}}$ crack surfaces remain in contact during shear load until the beginning of dynamic crack growth). The above explains gradual and nonlinear "flattening" of the dependence $\tau_{0}(P)$. Note that empirically derived expression (1) is a generalization of the conventional Griffith expression on different scale cracks being under the condition of confined shear loading.

Dependence of shear strength of intact interface $\tau_{\text {is }}$ on $\sigma_{\mathrm{n}}$ was derived analytically:

$$
\tau_{\text {is }}\left(\sigma_{\mathrm{n}}\right)=\sqrt{\frac{1}{3}\left[\frac{\left[\sigma_{\mathrm{c}}+a \sigma_{\mathrm{n}}(1+v) /(3(1-v))\right]^{2}}{b^{2}}-\sigma_{\mathrm{n}}^{2}\left(\frac{1-2 v}{1+v}\right)^{2}\right]},
$$

where $a=1.5\left(\sigma_{\mathrm{c}} / \sigma_{\mathrm{t}}-1\right)$ and $b=0.5\left(\sigma_{\mathrm{c}} / \sigma_{\mathrm{t}}+1\right)$ are parameters of applied fracture criterion of Drucker and Prager ( $\sigma_{\mathrm{c}}$ and $\sigma_{\mathrm{t}}$ are uniaxial compression strength and tensile strength of dry material), $v$ is a Poisson's ratio.

Analogous dependences for other constituents $\left(\alpha\right.$ and $\left.\tau_{\infty}\right)$ were derived by the analysis of numerical results:

$$
\begin{gathered}
\alpha\left(\sigma_{\mathrm{n}}\right)=\alpha_{0}-k\left(\sigma_{\mathrm{n}} / \tau_{\mathrm{is}}^{0}\right), \\
\tau_{\infty}\left(\sigma_{\mathrm{n}}\right)=C_{0} \tau_{\mathrm{is}}^{0}\left(\sigma_{\mathrm{n}} / \tau_{\mathrm{is}}^{0}\right)^{\gamma},
\end{gathered}
$$

where $\alpha_{0} \approx 0.55(1-v) \sqrt{1+v}$ [8], $k$ and $C_{0}$ are material-dependent parameters $\left(k \approx 0.75\right.$ and $C_{0} \approx 0.25$ for the considered material), $\gamma \approx 0.32, \tau_{\text {is }}^{0}$ is interface cohesion.

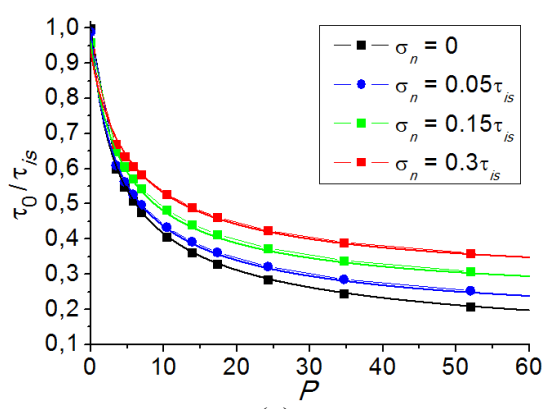

(a)

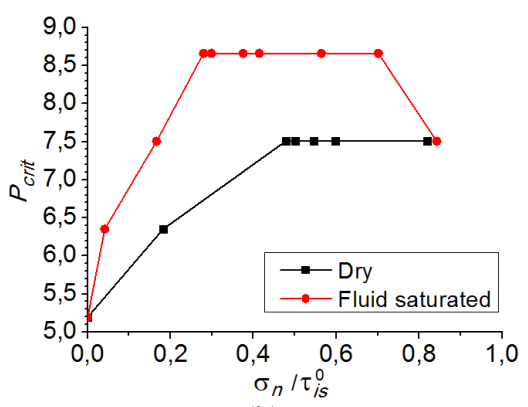

(b)

FIGURE 2. (a) The dependences of the shear strength $\tau_{0}$ of the slab with initial interface crack on $P$ at different $\sigma_{\mathrm{n}}$ (each series of points at certain value of $\sigma_{\mathrm{n}}$ is normalized to the value of shear strength of the intact interface $\tau_{\text {is }}$ at $\sigma_{\mathrm{n}}$ );

(b) the dependences of $P_{\text {crit }}$ on applied crack-normal stress $\sigma_{n}$ normalized to the value of interface cohesion 
Special study has shown that numerically derived dependence $\tau_{0}\left(P, \sigma_{\mathrm{n}}\right)$, which is a static characteristic of the shear crack, holds true both for dry and fluid-saturated nanoporous solids. At the same time dynamic properties of dry and fluid-saturated fluids may differ considerably. This concerns, in particular, the dependence of the critical value $P_{\text {crit }}$ of geometrical crack parameter $P$ (which is univocally related to the critical value of the shear strength of the slab with initial crack) on $\sigma_{\mathrm{n}}$. Note that the initial crack characterized by geometrical crack parameter $P$ is able to propagate in supershear regime only if the condition $P<P_{\text {crit }}$ is fulfilled (this is an equivalent of the energy condition $\left.\tau_{0}>\tau_{\text {crit }}\right)$. Figure $2 \mathrm{~b}$ shows dependences $P_{\text {crit }}\left(\sigma_{\mathrm{n}}\right)$ for dry and water-saturated model slabs. One can see that the critical value of dimensionless geometrical parameter in dry slab rapidly increases with crack-normal stress and then tends to saturation at the values of $\sigma_{\mathrm{n}}$ close to the half interface cohesion. At the same time analogous function for fluid-saturated sample is nonmonotonic. It has a cap shape and at high crack normal stresses converges to the same limiting value as in dry material. This difference is connected with the presence of strong compressive pressure wave ahead of the propagating crack tip in fluid-saturated solid. Highly compressed state of the material ahead of the crack impedes formation of a daughter crack and hence lead to a higher values of the critical crack parameter $P_{\text {crit }}$ at relatively low crack normal pressures.

Note that the most pronounced peculiarity the dynamics of supershear crack propagation in fluid saturated nanoporous material is the value of propagation velocity. Inhe considered fluid-saturated material it is more than $30 \%$ higher than in dry material (although the difference between the values of crack propagation velocity in the sub-Rayleigh regime does not exceed a few percent).

\section{CONCLUSION}

The study showed that the pore fluid in nanoporous brittle materials influences mainly the dynamic properties of longitudinal shear cracks, while static properties (including shear strength) of the material with initial crack are much less sensitive to the presence of pore fluid. The main peculiarity of the dynamic properties of the cracks in preliminary stressed fluid-saturated nanoporous material is nonmonotonic dependence of the critical value of dimensionless geometrical crack parameter (it characterizes limiting values of length and thickness of the cracks, that are capable to accelerate to intersonic velocity) on applied crack normal stress. This dependence has a maximum at relatively low values of crack normal stress and then decreases to limiting value, which coincide with limiting value for dry material. Note that the described features are specific for nanoporous brittle materials. In particular, the regularities of dynamic growth of shear cracks in the fluid saturated permeable materials with characteristic pore sizes amounting to a few tenths of a micrometer are close to the same for dry materials. The results confirm a complex and non-linear influence of fluid phase on dynamics of crack growth in brittle solids, which cannot be imitated using external mechanical loading of dry material [10, 11].

\section{ACKNOWLEDGMENTS}

The authors thank the Russian Science Foundation (Project 14-19-00718) for financial support.

\section{REFERENCES}

1. T.-F. Wong and P. Baud, J. Struct.Geol. 44, 25-53 (2012).

2. M. N. Bidgoli and L. Jing, Rock Mech. Rock. Eng. 48, 971-985 (2014).

3. Z. He, A. Kotousov, F. Berto, and R. Branco, Phys. Mesomech. 19(1), 6-20 (2016).

4. F. Barras, D. S. Kammer, P. H. Geubelle, and J. F. Molinary, Int. J. Fract. 189, 149-162 (2014).

5. I. Svetlizky, D. Pino Munoz, M. Radiguet, D. S. Kammer, J. F. Molinary, and J. Fineberg, Proc. Natl. Acad. Sci. USA 113, 542-547 (2016).

6. S. G. Psakh'e and K. P. Zol'nikov, Tech. Phys. Lett. 23, 555-556 (1997).

7. S. G. Psakhie, E. V. Shilko, M. V. Popov, and V. L. Popov, Phys. Rev. E 91, 063302-1-063302-6 (2015).

8. E. V. Shilko, S. G. Psakhie, and V. L. Popov, AIP Conf. Proc. 1683, 020209-1-020209-4 (2015).

9. S. G. Psakhie, E. V. Shilko, A. Yu. Smolin, A. V. Dimaki, A. I. Dmitriev, Ig. S. Konovalenko, S. V. Astafurov, and S. Zavsek, Phys. Mesomech. 14(5-6), 224-248 (2011).

10. E. Radi and B. Loret, Int. J. Fract. 147, 235-267 (2007).

11. N. Brantut and J. R. Rice, Geophys. Res. Lett. 38, L24314-1-L24314-6 (2011). 